\title{
CORRELAÇÃO DE LONGO ALCANCE TEMPORAL DA VELOCIDADE DO VENTO NOS MUNICÍPIOS DE CEARÁ-MIRIM E NATAL NO RIO GRANDE DO NORTE
}

\author{
N. F. LIMA ${ }^{*}$, M. A. C. Freire, J. J SANTOS e R. R. C. ALBUQUERQUE \\ Instituto Federal de Educação, Ciência e Tecnologia do Rio Grande do Norte \\ neilson.lima@ifrn.edu.br*
}

Submetido 24/12/2016 - Aceito 20/12/2017

DOI: $10.15628 /$ holos.2017.5491

\section{RESUMO}

A energia eólica é uma fonte natural de energia renovável e utilizada em diversos países para o abastecimento energético de residências, fábricas e empresas. Para os países que possuem hidrelétricas como a principal fonte geradora de energia, como o Brasil, por exemplo, a energia eólica é muito importante, porque ela não consome água, é renovável, limpa e não causa danos ambientais como outras fontes energéticas poluentes e sujas. Diversos estudos são realizados a fim de observar o comportamento do vento, em particular às correlações com outras variáveis como radiação solar, temperatura máxima ou mínima e umidade relativa do ar. Para fazerem inferência das observações do vento pesquisadores tem empregado diversas ferramentas estatísticas como médias móveis, média móvel
\end{abstract}

ponderada e suavização exponencial. Nosso interesse é verificar as correlações de curto ou longo alcance persistente/antipersistente em séries temporais de ventos dos municípios Natal e Ceará-Mirim. Para realizar o estudo da correlação do vento se aplicou os métodos estatísticos denominados Detrended Fluctuation Analysis (DFA) e Detrended Cross-Correlation Analysis -DCCA, isto é análise da flutuação sem tendências e análise da correlação cruzada sem tendências. Nesta pesquisa observou-se que a série temporal do vento tem uma forte correlação de longo alcance persistente, significando que valores altos de velocidade do vento tem maior probabilidade de ser seguido por valores altos; e, valores baixos na velocidade do vento tem maior probabilidade de ser seguido por valores baixos.

PALAVRAS-CHAVE: Correlação, probabilidade, série temporal, tendência.

\section{LONG-TERM CORRELATION OF WIND SPEED IN THE MUNICIPALITIES OF CEARÁ- MIRIM AND NATAL IN RIO GRANDE DO NORTE}

\begin{abstract}
Wind energy is a natural source of renewable energy and used in many countries for the energy supply of homes, factories and companies. For countries that own hydroelectric power as the main source of energy, such as Brazil, for example, wind energy is very important because it does not consume water, is renewable, clean and does not cause environmental damages like other polluting and dirty energy sources. Several studies are carried out to observe the wind behavior, in particular correlations with other variables such as solar radiation, maximum or minimum temperature and relative humidity. To make inference from wind observations researchers have employed various statistical tools such as moving averages, weighted moving average, exponential smoothing. Our interest is to verify the
\end{abstract}

correlations of short or long persistent / antipersistent in time series of winds of the municipalities and CearáMirim and Natal. In order to perform the wind correlation

study, statistical methods Detrended Fluctuation Analysis (DFA) e Detrended Cross-Correlation Analysis $D C C A$, le trendless fluctuation analysis and uncorrected cross correlation analysis. In this research it was observed that the wind time series has a strong persistent long-range correlation, meaning that high wind speed values are more likely to be followed by high values; And, low values in wind speed are more likely to be followed by low values.

KEYWORDS: Correlation, probability, time series, trend. 


\section{INTRODUÇÃO}

Na natureza existe diversos fenômenos complexos e difícil de analisar estatisticamente (JUNIOR, 2016), dentre eles se pode citar abalos sísmicos (Nóbrega \& Nóbrega, 2016), terremotos (SOUZA, BARROS, SILVEIRA, QUEIROZ \& SILVA, 2016), precipitação de chuva (ALVES, AZEVEDO \& FARIAS, 2016), velocidade e direção do vento (MUNHOZ \& GARCIA, 2008). O conjunto de observações ordenadas desses fenômenos são denominados de séries temporais, e muitas dessas séries temporais são não estacionárias, porque seus momentos estatísticos variam segundo a janela temporal escolhida na série.

Dentre os fenômenos naturais supra citados, destacamos, o vento. O vento é uma fonte de energia renovável e limpa, através do vento é possível gerar energia (eólica) capaz de atender casas, bairros, municípios, etc. O montante de energia disponível no vento varia segundo as estações do ano e as horas do dia, a topografia. Outra variável que influencia na geração de energia eólica é a rugosidade do solo, que têm grande influência na distribuição dos ventos (SOUZA, BARROS, SILVEIRA, QUEIROZ \& SILVA, 2016) e de sua velocidade. Além disso, a quantidade de energia eólica extraível numa região depende das características de desempenho, altura de operação e espaçamento horizontal dos sistemas de conversão de energia eólica instalados.

A crise energética atual preocupa estados e países do mundo, por isso governos e empresas estão investindo em fontes alternativas de energias, com destaque para a energia solar e eólica. A energia eólica tem benefícios como reduzir a emissão global de dióxido de carbono na atmosfera em mais de 10 bilhões de toneladas (LUIZ, CARDOSO \& RIBEIRO, 2016), porque será utilizada em substituição de fontes de energia poluentes; aumenta o número de empregos, é renovável e limpa.

Pesquisa científica vem aumentando no Brasil em relação às energias eólicas. Veem-se trabalhos de dissertações, teses e artigos científicos sendo publicados com as análises estatísticas de dados de ventos (ALVES, AZEVEDO \& FARIAS, 2016), (LUIZ, CARDOSO \& RIBEIRO, 2016).

Diante disto, nosso objetivo foi estudar as correlações de longo alcance das séries temporais da velocidade do vento no município de Natal e Ceará-Mirim no Rio Grande do Norte. Para este estudo foi usado um banco de dados de velocidade do vento disponibilizado pelo Instituto Nacional de Meteorologia-INMET no endereço http://www.inmet.gov.br/portal/. Para estudar as correlações foi aplicado o método estatístico Detrended Fluctuation Analysis (DFA), isto é, análise da flutuação sem tendência, desenvolvido por Peng em 1994 e o Detrended CrossCorrelation Analysis -DCCA, ou seja, análise da correlação cruzada sem tendência, desenvolvido por Podoboniq em 2008. O DFA e o DCCA faz análise de séries temporais não estacionárias, e através destes métodos é possível analisar se existe correlação de longo alcance ou se não existe correlação. Existindo a correlação, é possível observar se a correlação é positiva, negativa, persistente ou antipersistente, de curto alcance ou de logo alcance.

Nesta pesquisa, observou-se que a velocidade do vento nos municípios citados são de longo alcance persistente, indicando que velocidades altas de ventos tem maior probabilidade de ser seguidas por velocidades altas de ventos, de forma equivalente, velocidades baixas de vento 
tem maior probabilidade de ser seguida por velocidades baixas de vento. Assim este resultado é importante para a possibilidade de implantação de parque eólicos, porque já fornece uma análise essencial do comportamento da velocidade média diária do vento

\section{REVISÃO BIBLIOGRÁFICA}

$\mathrm{Na}$ literatura muitos trabalhos abordam a velocidade do vento, pesquisadores tem estudados velocidade, direção, distribuição de probabilidade (Normal, Gumbel, Fréchet, Weibull, LogNormal e Pearson 3) (DO NASCIMENTO, LUCIO \& JUNIOR, 2016), frequência relativa, distribuição a prioi e a posteriori (SANSIGOLO, 2005). O interesse de tais pesquisadores é analisar estatisticamente a fim de fazer predição e previsões do comportamento da velocidade do vento.

Diversos métodos estatísticos são empregados para analisar as correlações na série temporal da velocidade do vento. Dentre os métodos estatísticos, pode-se citar a estatística de Hurst. Harold E. Hurst (1880 - 1978) (DE ARAUJO, STOSIC, STOSIC \& DEZOTTI, 2015) foi um hidrólogo que passou parte de sua vida dedicando-se a descrição dos problemas relacionados aos reservatórios de água do rio Nilo. No decorrer de seus estudos Hurst percebeu que as vazões e enchentes do rio Nilo caracterizavam-se como um processo estocástico. Com objetivo de descrever os problemas de dimensionamento das represas foi proposto por Hurst (1951) , a análise estatística R/S , descrita em detalhes no livro Long-Term Storage: An Experimental Study (DE ARAUJO, STOSIC, STOSIC \& DEZOTTI, 2015).

A estatística R/S (PENG, BULDYREV, HAVLIN, SIMONS, STANLEY \& GOLDBERGER, 1994) consistia na mensuração dos volumes máximos e mínimos do reservatório (amplitude) e no cálculo do desvio-padrão dos fluxos de água em um período de tempo $\tau$, de modo que $R / S$ seria a razão entre a amplitude $\left(R_{\tau}\right)$ e o desvio padrão $\left(S_{\tau}\right)$ das vazões de água, que é um valor adimensional. Hurst, ao realizar essa estatística para diversos períodos de tempo, através desse modelo, constatou que a estatística R/S apresentava uma função de relacionamento com o número de observações do cálculo, inclusive para outros fenômenos naturais.

Os valores do expoente de Hurst tem domínio de variação entre 0 e 1 . Se o coeficiente de Hurst for igual a 0,5 a série é denotada como browniana ou descorrelacionada (aleatório). Quando $\mathrm{H}$ estiver compreendido $0,5<\mathrm{H} \leq 1$ a série é dita persistente (GVOZDANOVIC, PODOBNIK, WANG \& STANLEY, (2012). Para $\mathrm{H}$ variando de $0 \leq \mathrm{H}<0,5$, a série pode-se inferir que a série apresenta comportamento de antipersistência. A estatística $R / S$ originalmente aplicada em hidrologia tem aplicação em diversas áreas da ciência como, por exemplo, estudo de temperaturas globais, volatilidade de ativos financeiros, velocidade e direção de ventos, umidade relativa do ar, radiação solar entre outras. Apesar da robustez matemática o modelo de Hurst necessita de filtragem antes da aplicação a fim de retirar possíveis tendências.

Outro método estatístico para o estuda da velocidade do vento é o método Detrended Fluctuation Analysis (DFA) (GVOZDANOVIC, PODOBNIK, WANG \& STANLEY, (2012) desenvolvido por Peng et.al [26] para quantificar correlações em séries temporais não estacionárias e o método Detrended Cross-Correlation Analysis -DCCA (GVOZDANOVIC, PODOBNIK, WANG \& STANLEY, (2012). Estes métodos foram utilizados para análises de correlações de sequência gênicas de DNA, correlações de séries financeiras, climatologia, meteorologia, precipitação pluviométrica, entre outros. Os métodos DFA e DCCA evitam cometer erros de diagnóstico de correlações persistente ou antipersistente. 
No ano de 2015 o artigo intitulado Long-term correlations and cross-correlations in wind speed and solar radiation temporal series from Fernando de Noronha Island, Brazil, de autoria de Sales et al.[ DOS ANJOS, PRISCILLA SALES ET AL, 2015] foi publicado na revista Physica $A$. Neste artigo consta um estudo da correlação da velocidade do vento na Ilha de Fernando de Noronha, onde foi observada a correlação de curto ou longo alcance persistente ou antipersistente conforme a janela temporal considerada.

\section{METODOLOGIA}

As séries temporais de velocidade de vento são complexas de analisar devido seu comportamento aleatório e não estacionário, e, fazer estudos sobre previsão, correlações de curto ou longo alcance temporal não é uma tarefa simples. A fim de estudar as autocorrelações (LI, NISHIMURA \& MEN, 2016) e as correlações cruzadas da velocidade do vento dos municípios Ceará-Mirim e Natal (Figura 1), localizados no estado do Rio Grande do Norte, Brasil, foram aplicados dois métodos estatísticos: Detrended Fluctuation Analysis (DFA) e Detrended CrossCorrelation Analysis -DCCA.
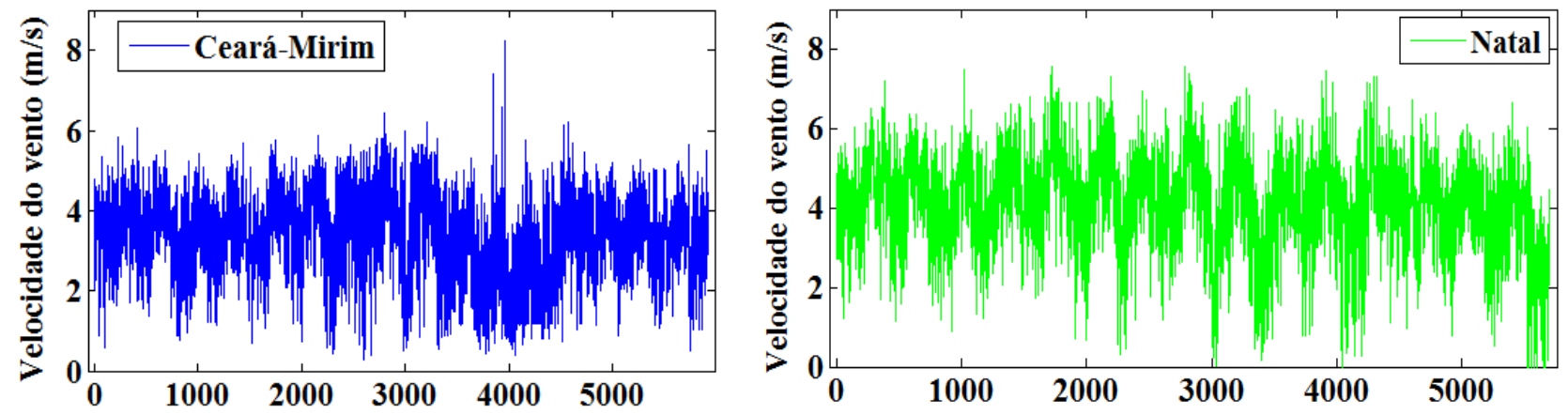

Figura 1: Serie temporal da velocidade $(\mathrm{m} / \mathrm{s})$ do vento dos municípios Ceará-Mirim e Natal no período de 01 de Fevereiro de 2000 até 10 de Julho de 2016.

Os dados desta pesquisa foram disponibilizados pelo Instituto Nacional de Meteorologia INMET no endereço http://www.inmet.gov.br/portal/. Tomou-se os registros diários da velocidade do vento $(\mathrm{m} / \mathrm{s})$ no período de 01 de Fevereiro de 2000 até 10 de Julho de 2016.

De posse desses dados se formou uma nova série temporal dada pela Equação (1)

(1)

$$
x(i)=\frac{\mid \log \left(z_{i+\Delta i)}-\log \left(z_{i}\right) \mid\right.}{\sigma}
$$

em que $z_{i}$ representa as observações diárias no índice $i=1, \ldots, N$, onde $N$ indica a quantidade de observações diárias, $\Delta i$ é o intervalo das observações, neste caso $\Delta i=1 \mathrm{dia}$, e $\sigma$ é o desviopadrão dos valores absolutos da série de retorno dos logaritmos.

\subsection{Detrended Fluctuation Analysis (DFA)}

Toma-se a série temporal $x(i)$, onde $i=1,2, \ldots, N$, sendo $N$ o cumprimento ou tamanho da série temporal; integra-se o sinal $x(i)$ e obtêm-se $y(k)$, Equação (2), 


$$
y(k)=\sum x(i)-\langle x\rangle
$$

onde $\langle x\rangle$ é a média do sinal $x(i)$, em seguida divide-se o sinal integrado $y(k)$ em caixas de mesmo tamanho $n$ justapostos, então ajustamos $y(k)$ por meio de uma função polinomial de grau I (que no nosso caso ajuste foi feito por uma função polinomial de grau 1, e uma função de grau 2, função quadrática (PENG, BULDYREV, HAVLIN, SIMONS, STANLEY \& GOLDBERGER, 1994), (GVOZDANOVIC, PODOBNIK, WANG \& STANLEY, (2012), que representa a tendência na caixa específica; continuando, o sinal $y(k)$ é subtraindo de $y_{n}(k)$ que representa a tendência local em cada caixa. Para quantificar as flutuações foi usada a função de flutuação na Equação (3) em todas as caixas para cada $n$

$$
F(n)=\sqrt{\frac{1}{N} \sum\left[y(k)-y_{n}(k)\right]^{2}}
$$

quando há lei de potência neste cálculo interativo, então $F(n)$ segue uma lei de potência, do tipo $F(n) \propto n^{\alpha}$, em que $\alpha \approx H$ (expoente de Hurst) (DOS ANJOS, DA SILVA, STOSIC \& STOSIC, 2015). A persistência nestas séries temporais pode ser caracterizada pela função de auto correlação $C(t)$, em que $t$ é o tempo entre as observações; esta função decai como $C(t) \sim t^{-\gamma}$ (lei de potência). 0 expoente $\gamma$ está relacionado com o expoente $\alpha$ do seguinte modo $\gamma=2-2 \alpha$.

Para obter o expoente $\alpha$ foi calculado o coeficiente angular da reta $\log (F(n))$ versus $\log (n)$. Assim, se $0<\alpha<0,5$ (auto afinidade) as autocorrelações da série original são antipersistentes; se $0,5<\alpha \leq 1$ as autocorrelações são persistentes; se $\alpha=0,5$ a série não apresenta correlação (ruído branco), e se $\alpha>1$ as correlações existem, mas não apresentam lei de potência (DOS ANJOS, DA SILVA, STOSIC \& STOSIC, 2015), (LI, NISHIMURA \& MEN, 2016).

\subsection{Detrended Cross-Correlation Analysis -DCCA}

O método DCCA (DOS ANJOS, DA SILVA, STOSIC \& STOSIC, 2015), (GVOZDANOVIC, PODOBNIK, WANG \& STANLEY, 2012), (PODOBNIK, \& STANLEY, 2008) procede do seguinte modo: toma-se duas séries temporais $\left\{y_{i}\right\}$ e $\left\{y_{i}^{\prime}\right\}$ de tamanho $N$, integra-se essas séries pelas expressões $R_{k}=y_{1}+y_{2}+\cdots+y_{k}$ e $R_{k}^{\prime}{ }_{k}=y_{1}^{\prime}+y_{2}^{\prime}+\cdots+y^{\prime}{ }_{k}$, com $k=1,2, \ldots, N$; divide-se os sinais $R_{k}$ e $R^{\prime}{ }_{k}$ em $N-n$ caixas sobrepostas contendo $n+1$ pontos ou valores; para cada caixa das séries temporais $\left\{y_{i}\right\}$ e $\left\{y_{i}^{\prime}\right\}$ (que inicia em $i$ e termina em $i+n$ ) foi definida a tendência local ( $\widetilde{R_{k, l}}$ e $\widetilde{R_{k, l}^{\prime}}$ respectivamente) como sendo a ordenada do ajuste linear dos mínimos quadrados da série integrada. Agora, retira-se a tendência para cada intervalo e se calcula a covariância dos resíduos para os intervalos

$$
f_{D C C A}^{2}(n, i) \equiv \frac{1}{n-1} \sum_{k=i}^{i+n}\left(R_{k}-\widetilde{R_{k, l}}\right)\left(R^{\prime}{ }_{k}-\widetilde{R_{k, l}^{\prime}}\right)
$$

Em seguida calcula-se a covariância sem tendência somando todos os $N-n$ intervalos sobrepostos de tamanho $n+1$ pela expressão:

$$
F_{D C C A}^{2}(n, i) \equiv \frac{1}{N-n} \sum_{i=1}^{N-n} f_{D C C A}^{2}(n, i) .
$$


Repetindo-se esse cálculo interativamente para diversos tamanhos de $n$, pode-se observar a relação entre as flutuações $F_{D C C A}^{2}(n, i)$ e o cumprimento do intervalo $n$. Se houver correlação cruzada de longo alcance, então $F_{D C C A}^{2}(n, i)$ versus $n$ segue uma lei de potência $\left(F_{D C C A}^{2}(n, i) \propto n^{\lambda}\right)$. Pode-se obter o expoente $\lambda$ (auto afinidade) como o coeficiente angular da reta $\log (F(n))$ versus $\log (n)$, e sua interpretação é semelhante ao expoente $\alpha$ do DFA (GVOZDANOVIC, PODOBNIK, WANG \& STANLEY, (2012), isto é, se $0<\lambda<0,5$ (auto afinidade) as autocorrelações da série original são antipersistentes; se $0,5<\lambda \leq 1$ as autocorrelações são persistentes; se $\lambda=0,5$ a série não apresenta correlação (ruído branco), e se $\lambda>1$ as correlações existem, mas não apresentam lei de potência.

\section{RESULTADOS E DISCUSSÕES}

Os métodos estatísticos DFA e DCCA foram aplicados as séries temporais diárias da velocidade média do vento dos municípios Ceará-Mirim e Natal no Rio Grande do Norte. Na Figura 2 se ver os resultados usando o DFA para Ceará-Mirim e na Figura 3 para Natal. Para os dois municípios, retirou-se as tendências linear e quadrática.

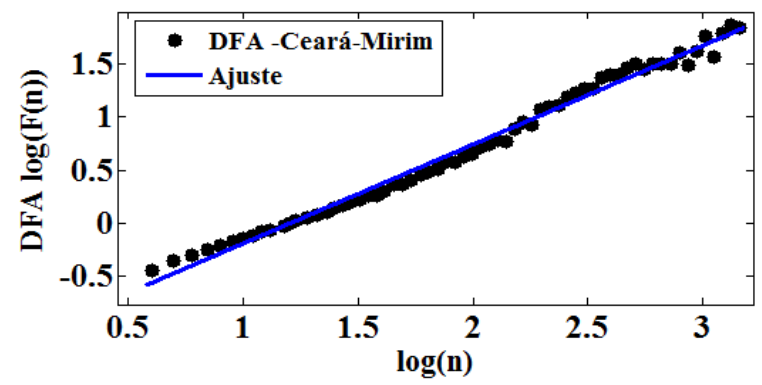

(a)

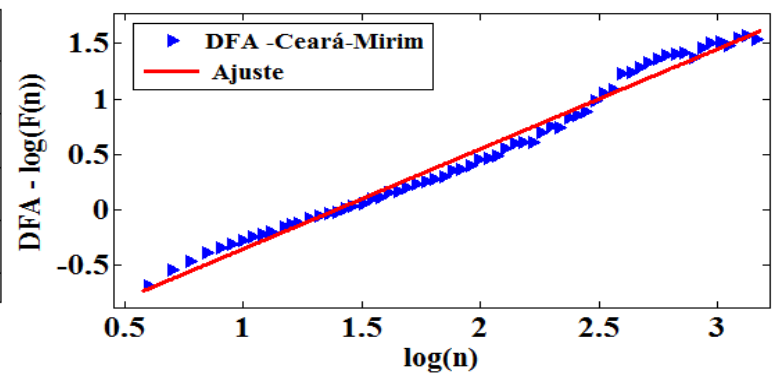

(b)

Figura 2: Nesta Figura está o resultado da análise da auto correlação parcial das flutuações sem tendência -DFA da velocidade do vento do município Ceará-Mirim/RN. Na Figura 2(a) e 2(b) vê-se o resultado da análise após a retirada das tendências linear e quadrática, respectivamente, da série temporal $\boldsymbol{y}(\boldsymbol{k})$ na escala log-log.

Na Figura 2(a), as bolinhas pretas representam a flutuação $(\log (F(n))$ e, a reta de cor azul a tendência dessas flutuações. Retirou-se das caixas justapostas da série temporal integrada a tendência linear (Figura 2(a)). Na aplicação do DFA, retirando a tendência linear, o valor de $\alpha$ foi igual a $0.9297 \pm 0.0262(\alpha=0.9297 \pm 0.0262)$, indicando que velocidades médias diárias do vento altas tem maior probabilidade de ser seguida por velocidades altas e, velocidades baixas do vento tem maior probabilidade de ser seguida por velocidades baixas (PODOBNIK, \& STANLEY, 2008). Observe que a auto correlação parcial é forte, porque o seu valor está muito próximo de 1.

Na Figura 2(b), os pequenos triângulos azuis ilustram a flutuação e, a reta de cor vermelha a tendência linear. Na aplicação do DFA, para este caso, foi retirada a tendência quadrática da série integrada, e foi obtido para o expoente da lei de potência $F(n) \propto n^{\alpha}$ o valor de $0.9003 \pm$ 0.0310 ( $\alpha=0.9003 \pm 0.0310)$, indicando uma correlação persistente, isto é, que valores altos da velocidade do vento tem maior probabilidade de serem seguidos por valores altos.

Na Figura 3, de forma semelhante à Figura 2, está o resultado da aplicação do DFA para a série de velocidade do vento de Natal. Retirando-se a tendência linear (Figura 3(a)) o expoente de auto correlação foi $\alpha=0.9017 \pm 0.0258$, já retirando a tendência quadrática (Figura 3(b)), o expoente $\alpha=0.9237 \pm 0.0256$. Nos dois casos, linear e quadrático, as correlações na série são 
persistentes. Isto significa que se observarmos as velocidades dos ventos em janelas temporais vizinhas, então se pode inferir que quando a velocidade do vento está aumentando para uma janela temporal de tamanho $k$, esta velocidade do vento terá maior probabilidade de aumentar para uma janela temporal vizinha $k+\Delta k$.

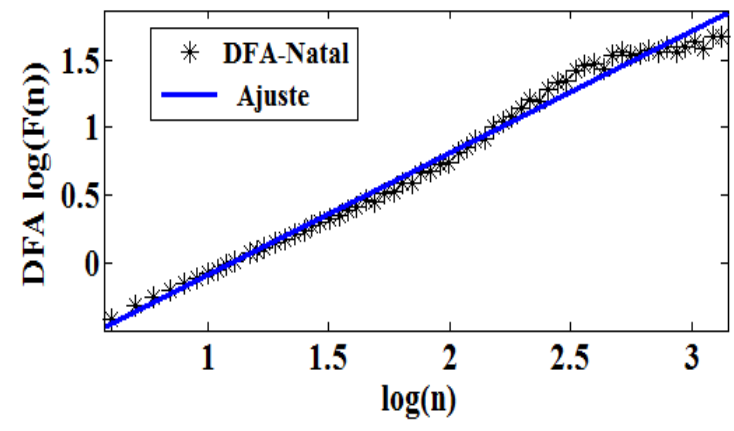

(a)

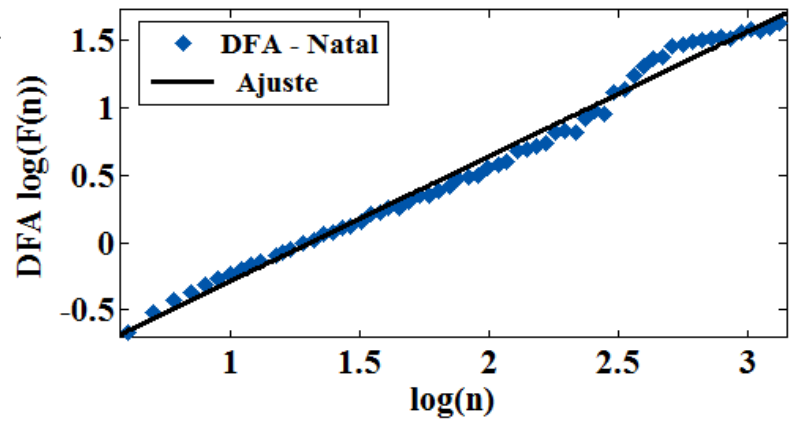

(b)

Figura 3: Nesta Figura está o resultado da análise da auto correlação parcial das flutuações sem tendência -DFA da velocidade do vento do município Natal/RN. Na Figura 2(a) e 2(b) vê-se o resultado da análise após a retirada das tendências linear e quadrática, respectivamente.

Na Figura 4 está ilustrado os resultados da aplicação do DCCA entre as séries temporais da velocidade do vento dos municípios Natal e Ceará-Mirim. De forma semelhante ao que foi feito com o DFA, também foi retirado as tendências linear (Figura 4(a)) e quadrática (Figura 4(b)).

A correlação cruzada entre a velocidades do vento dos municípios Natal e Ceará-Mirim foi forte e persistente, porque teve um expoente $\lambda=0.7081 \pm 0.0228$ para o caso linear, e, $\lambda=0.6875 \pm 0.0203$ retirando a tendência quadrática (Tabela 1), (PODOBNIK, \& STANLEY, 2008). Infere-se deste resultado que há uma correlação positiva entre as velocidades do vento destes municípios, assim velocidades altas de vento em Natal implica em uma maior probabilidade de velocidade alta de vento em Ceará-Mirim, de forma equivalente, velocidade baixa de vento em Natal implica em uma maior probabilidade da velocidade baixa do vento em Ceará-Mirim.

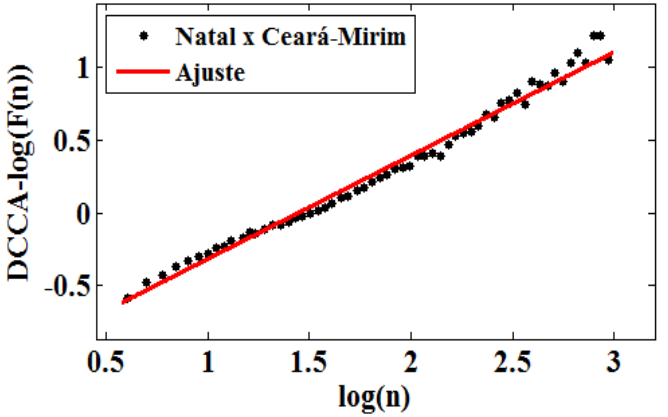

(a)

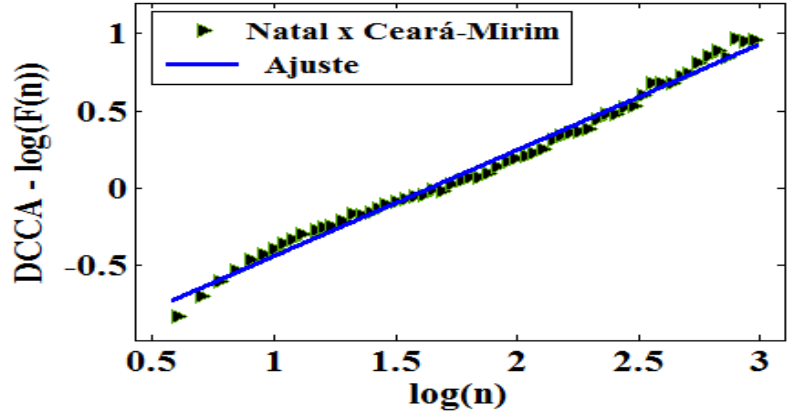

(b)

Figura 4: Nesta Figura está o resultado da análise das correlações cruzadas das flutuações sem tendência-DCCA da velocidade do vento entre os municípios Natal e Ceará-Mirim. Na Figura 2(a) e 2(b) vê-se o resultado da análise após a retirada das tendências linear e quadrática, respectivamente, na escala log-log. 
Tabela 1: Resultados do expoente DFA e DCCA para os municípios de Ceará-Mirim e Natal.

\begin{tabular}{c|c|c|c|c}
\hline & \multicolumn{2}{|c|}{ DFA } & \multicolumn{2}{c}{ DCCA } \\
\hline \multirow{3}{*}{ Ceará-Mirim } & Linear & Quadrática & Linear & Quadrática \\
Natal & $0.9297 \pm 0.0262$ & $0.9003 \pm 0.0310$ & & \\
& $0.9017 \pm 0.0258$ & $0.9237 \pm 0.0256$ & $0.7081 \pm 0.0228$ & $0.6875 \pm 0.0203$ \\
\hline
\end{tabular}

\section{CONCLUSÃO}

$\mathrm{Na}$ análise dos tipos de correlação da velocidade do vento dos municípios Natal e CearáMirim não houve correlação antipersistente, nem descorrelacionada (ruído branco), mas apenas correlação persistente (Tabela 1), porque os valores dos expoentes $(\alpha-D F A)$ e $(\lambda-D C C A)$ que indicam o tipo de correlação foram maiores que meio (0.5). Vale salientar que os valores de $0.5<\alpha \leq 1$ e $0.5<\lambda \leq 1$ não exclui a probabilidade de valores baixos na velocidade do vento ser seguido por valores altos de velocidade de vento, mas indica que essa probabilidade tende a ser pequena.

Observou-se que as diferenças entre as tendências linear e quadrática (Tabela 1) foram pequenas para os dois municípios. Para Ceará-Mirim a diferença foi de 0.0924 (3.16\%), enquanto para Natal foi de 0.022 (2.44\%) considerando o DFA. Já para o DCCA, a diferença foi 0.0206 (3\%), também pequena.

\section{REFERÊNCIA}

ALVES, T. L. B., AZEVEDO, P. V., \& FARIAS, A. A. (2016). Comportamento da precipitação pluvial e sua relação com o relevo nas microrregiões do Cariri Oriental e Ocidental do estado da Paraíba (Rainfall behavior rain and its relationship with relief in the Regions Cariri of Eastern and Western state of Paraíba). Revista Brasileira de Geografia Física, 8(6), 1601-1614.

DE ARAUJO, A. J., STOSIC, T., STOSIC, B., \& DEZOTTI, C. H. (2015). Correlações de longo alcance em séries temporais de velocidade do vento no Nordeste. Sigmae, 2(3), 81-84.

DO NASCIMENTO CAMELO, H., LUCIO, P. S., \& JUNIOR, J. B. V. L. (2016). Modelagem de média mensal de velocidade do vento para região litorânea no nordeste Brasileiro através do método aditivo HoltWinters com vias a previsão de geração eólica. Revista Brasileira de Energias Renováveis, 5(4).

DOS ANJOS, P. S., DA SILVA, A. S. A., STOŠIĆ, B., \& STOŠIĆ, T. (2015). Long-term correlations and cross-correlations in wind speed and solar radiation temporal series from Fernando de Noronha Island, Brazil. Physica A: Statistical Mechanics and its Applications, 424, 90-96.

GVOZDANOVIC, I., PODOBNIK, B., WANG, D., \& STANLEY, H. E. (2012). 1/f behavior in crosscorrelations between absolute returns in a US market. Physica A: Statistical Mechanics and its Applications, 391(9), 2860-2866.

JÚNIOR, J. M. C. (2016). Strawberry cultivars: Knowing to expand and reduce the environmental impacts| Cultivares de morango: conhecer para expandir e reduzir os impactos ambientais. Revista Geama, 5(1), 138-147. 
LI, D., NISHIMURA, Y., \& MEN, M. (2016). Why the long-term auto-correlation has not been eliminated by arbitragers: Evidences from NYMEX. Energy Economics, 59, 167-178.

LUIZ, G. C., CARDOSO, H. C., \& RIBEIRO, L. L. (2016). Aplicação do teste sazonal de mann kendall na análise de tendência da temperatura e umidade relativa do ar-goiâniago: série histórica 1961 A 2008. Revista geonorte, 3(8), 414-427.

MUNHOZ, F. C., \& GARCIA, A. (2008). Caracterização da velocidade e direção predominante dos ventos para a localidade de Ituverava, SP. Revista Brasileira de Meteorologia, 23(1), 30-34.

NOBREGA, P. G. B., \& NOBREGA, S. H. S. (2016). Perigo sísmico no brasil e a responsabilidade da engenharia de estruturas. HOLOS, 4, 162-175.

PENG, C. K., BULdYREV, S. V., HAVliN, S., SIMONS, M., STANLEY, H. E., \& GOLDBERGER, A. L. (1994). Mosaic organization of DNA nucleotides. Physical review e, 49(2), 1685.

PODOBNIK, B., \& STANLEY, H. E. (2008). Detrended cross-correlation analysis: a new method for analyzing two nonstationary time series. Physical review letters, 100(8), 084102.

SANSIGOLO, C. A. (2005). Distribuições de probabilidade de velocidade e potência do vento. Revista Brasileira de Meteorologia, 20(2), 207-214.

SOUZA, J. L., BARROS, J. D., SILVEIRA, A. H. D. M., QUEIROZ, A. F. S., \& SILVA, F. M. (2016). Probabilidade de eventos bioclimáticos em Natal/RN. Revista do CERES, 1(2), 241-244. 